\section{Be sweet to toddlers during needles: pilot randomized controlled trial of sucrose compared to placebo}

\author{
Denise Harrison, ${ }^{1,2}$ Jessica Reszel, ${ }^{3}$ \\ Nick Barrowman, ${ }^{3}$ Brenda Martelli, ${ }^{4}$ \\ Diane Sharp, ${ }^{5}$ Régis Vaillancourt ${ }^{6}$ \\ ${ }^{1}$ Children's Hospital of Eastern Ontario; \\ ${ }^{2}$ School of Nursing, University of Ottawa; \\ ${ }^{3}$ Children's Hospital of Eastern Ontario \\ Research Institute; ${ }^{4}$ Integrated Pain \\ Services, Children's Hospital of Eastern \\ Ontario; ${ }^{5}$ Corporate Patient Services, \\ Children's Hospital of Eastern Ontario; \\ 'Pharmacy, Children's Hospital of Eastern \\ Ontario, Ottawa, Canada
}

\section{Abstract}

Sweet solutions reduce procedural pain in infants but there is uncertainty about effectiveness beyond infancy. A blinded pilot randomized controlled trial (RCT) was conducted to inform a full-scale RCT. Hospitalized children aged 1236 months were randomized to $24 \%$ sucrose or water prior to and during venipuncture. Primary outcomes were crying time and FLACC (Face, Legs, Activity, Cry, Consolability) scores. Secondary outcomes parental report of child's pain, parental perception of effectiveness and nurse's score of child's compliance with study solution. Twenty-one children were studied. There were no differences in pain outcomes between groups. Median FLACC scores at time of needle insertion were high (8/10), and mean crying time during procedure was $61 \%$. Most parents $(n=17)$ would use the same treatment next time and most $(n=17)$ toddlers were compliant with receiving the solutions. The pilot RCT informed the conduct of a future full scale RCT in terms of feasibility, acceptability, data collection, data analysis and sample size estimation.

\section{Introduction}

Venous access procedures for blood sampling (venipuncture) and intravenous (IV) catheter insertion are the most commonly performed painful procedures that hospitalized children undergo. ${ }^{1}$ Most children are afraid of needles, and rate needles as being the most distressing aspect of hospitalization. ${ }^{2}$ Children's distress has a negative impact on their parents and increases the risk of avoidance of needles for immunizations and med- ical care. ${ }^{3}$ Extensive high quality evidence from randomized controlled trials (RCTs) and systematic reviews demonstrate analgesic effects of sweet solutions in infants undergoing needle related procedures. ${ }^{4}$ It is uncertain if the same analgesic effects persist beyond infancy. Only two trials in a Cochrane systematic review of sweet solutions for procedural pain reduction in children included young children up to three years of age and results of these trials were conflicting. ${ }^{5}$ Both RCTs evaluated sucrose during immunization. Venipuncture is a more prolonged procedure involving tourniquet placement, needle insertion and adjustment and withdrawal of blood. The study's objective was therefore to conduct a pilot RCT of sucrose compared to placebo during venipuncture in young children to inform a full-scale RCT.

\section{Materials and Methods}

\section{Trial design}

A parallel, blinded, two-armed pilot RCT (http://www.anzctr.org.au; ACTRN126100 00355077). An allocation ratio of 1:1 was used.

\section{Participants}

Inpatients in two medical/surgical units at a tertiary pediatric hospital in Canada were recruited daily by study research assistants. Eligibility criteria: aged 12 to 36 months; undergoing medically required venous blood sampling; eligible to receive sucrose as per the hospital's sucrose policy; parent/guardians' understanding of English or French. Exclusion criteria: administration of muscle relaxants, opioid analgesics or sedatives in the $24 \mathrm{~h}$ prior to data collection; already consuming sweet fluids or foods; if the mother wished to breastfeed during the procedure; if the child had a known or suspected fructose intolerance or sensitivity to Ametop.

Participants were randomized by computergenerated random numbers in randomly-varying blocks of four and six, prepared by a statistician. The hospital's pharmacy department prepared the study solutions in identical syringes, as per the randomization codes and numbered sequentially. As both solutions are clear and colorless, concealment of allocation was maintained for the parents, bedside nurse, study research assistant, and outcome assessors. A sample size of $30 ; 15$ per group, was considered sufficient to establish baseline data to conduct future sample size estimation.

\section{Interventions}

Standard of care for all children included topical Ametop applied at least 40 min prior to commencing the venipuncture procedure,
Correspondence: Denise Harrison, 401 Smyth Road, Ottawa 0N, K1H 8L1, Canada.

Tel.: 613.737 .7600 ext. 4140.

E-mail: dharrison@cheo.on.ca

Key words: Pain; painful; children; sucrose; venipuncture.

Acknowledgements: Yehudis Stokes and Kowsar Abdulla for their assistance with data collection; Marina Walcer and Margot Thomas for their assistance with coding of pain scores and cry time.

Contributions: DH, NB, BM, DS, RV, conception and design of the study; JR, data collection; JR and NB, data analysis; DH and JR, writing of the manuscript; all authors reviewed and approved the final manuscript.

Funding: this study was funded by a Children's Hospital of Eastern Ontario Research Institute Internal Grant.

Conflict of interests: no conflicts of interest to declare for any authors.

Ethics approval: Children's Hospital of Eastern Ontario Research Ethics Board (\#11/20E).

Received for publication: 6 April 2015.

Revision received: 6 May 2015.

Accepted for publication: 7 May 2015.

This work is licensed under a Creative Commons Attribution NonCommercial 3.0 License (CC BYNC 3.0).

@C Copyright D. Harrison et al., 2015

Licensee PAGEPress, Italy

Nursing Reports 2015; 5:5218

doi:10.4081/nursrep.2015.5218

parental or staff holding, and age appropriate distraction. In addition, children in the intervention group received $24 \%$ oral sucrose and the control group received sterile water. Both study solutions were administered onto the anterior portion of the child's tongue in 0.25 $\mathrm{mL}$ aliquots two minutes prior to the needle insertion, immediately prior to the needle insertion, and every two minutes until the procedure was complete, up to a maximum of 3 mL. Study solutions were administered by the child's bedside nurse.

To ensure standardization of the blood collection procedure, all venipuncture procedures were performed by the hospital's Vascular Access Team. Approval for the study was obtained from the hospital's Research Ethics Board. All procedures were video-taped for later pain scoring.

\section{Outcomes}

Primary outcomes: 10-point pain scale FLACC (Face, Legs, Activity, Cry, Consolability) 
and cry duration. FLACC scores were completed before, during, and after the procedure and crying time was measured from administration of first study solution dose to $30 \mathrm{~s}$ following completion of procedure. Assessments were performed from video-recordings by coders external to the study, and blinded to the allocation of study solutions.

Secondary outcomes: parental report using a $10 \mathrm{~cm}$ visual analogue scale (VAS), parental perception of effectiveness of the treatment, and the nurse's perception of the child's compliance with taking the study solution.

\section{Data analysis}

Intention to treat analysis of pilot data was used. FLACC scores, crying time and parental report of the $10 \mathrm{~cm}$ VAS were summarized using mean, standard deviation, and Two-sample Student's $t$-test if normally distributed, and median, interquartile range (IQR) and Mann Whitney test if non-normally distributed. If one of the components of a FLACC score was missing, the score was included and data was imputed using the calculation - (FLACC score/4) $x$ 5. If two or more components were missing, the score for that time point was omitted from analysis. Secondary outcomes were reported as frequencies and analyzed using Fisher's exact test. A sample size calculation for a future full-scale RCT was conducted.

\section{Results}

Between August 2012 and July 2013, 21 chil- dren were randomized and studied; 11 received sucrose and 10 received water (Figure 1). Recruitment was stopped before the anticipated 30 children were studied until authorization could be obtained from Health Canada for our institutional manufactured sucrose product (routinely used in clinical care). However, this approval was unable to be obtained in a timely manner.

The mean age of the sample was 22.2 months. For two-thirds of the children this was their first hospitalization (66.7\%), and most had received sucrose previously (71.4\%). There were no significant differences in demographic characteristics between the groups. Most children were laid flat during the venipuncture procedure $(81 \%)$ with a median of $3(\mathrm{IQR}=1)$ people holding the child down. The median duration of the venipuncture procedure was $250 \mathrm{~s}(\mathrm{IQR}=171)$. Primary and secondary outcome measures are summarized in Table 1.

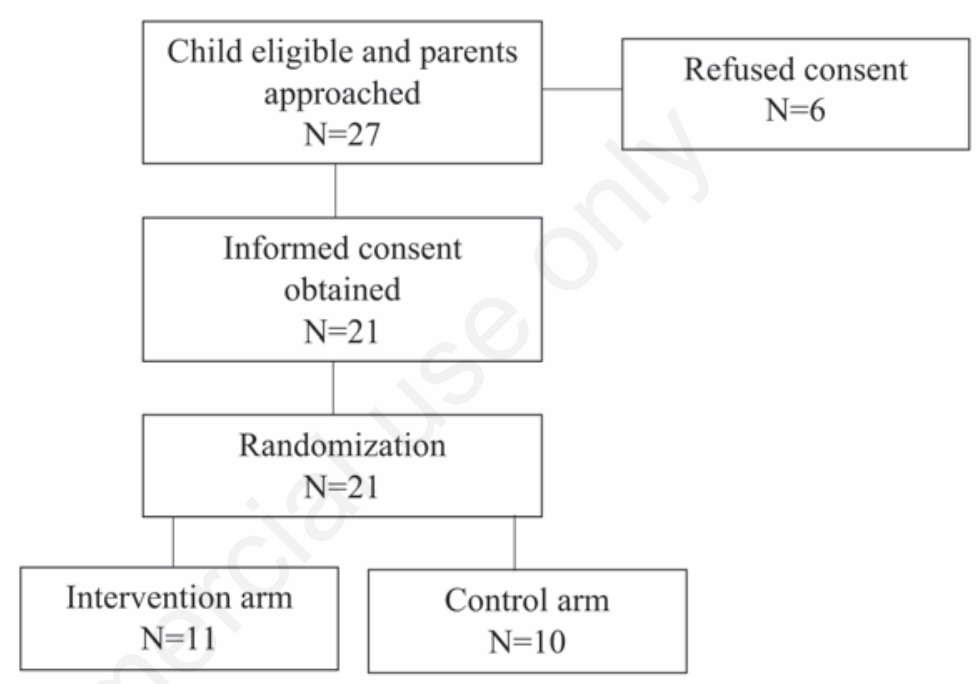

Table 1. Summary of results.

\begin{tabular}{|c|c|c|c|c|}
\hline & $\begin{array}{l}\text { Total } \\
(n=21)\end{array}$ & $\begin{array}{l}\text { Intervention } \\
\quad(\mathrm{n}=11)\end{array}$ & $\begin{array}{l}\text { Control } \\
(\mathrm{n}=10)\end{array}$ & $\mathbf{P}$ \\
\hline FLACC - prior to the administration of the study solution (median, IQR) & $5(7.5)$ & $6(7)$ & $3.5(8.25)$ & $0.557^{*}$ \\
\hline FLACC - immediately following the first dose of the study solution (median, IQR) & $3(7)$ & $7(7)$ & $1.5(8.25)$ & $0.282 *$ \\
\hline FLACC - upon placement of the tourniquet (median, IQR) & $8(5)$ & $8(2)$ & $6.5(5.75)$ & $0.654^{*}$ \\
\hline FLACC - at the time of first needle insertion (median, IQR) & $8(4)$ & $8(2)$ & $8.375(5.25)$ & $0.705^{*}$ \\
\hline FLACC - upon removal of the needle (median, IQR) & $8(5.5)$ & $8(7)$ & $7.5(5.25)$ & $0.863^{*}$ \\
\hline FLACC - one minute after removal of needle (median, IQR) & $3.75(5.63)$ & $5(6)$ & $2(5.69)$ & $0.468^{*}$ \\
\hline Total crying time in seconds (mean, SD) & $183.76(111.16)$ & $197.27(128.88)$ & $168.9(92.40)$ & $0.573^{\circ}$ \\
\hline Proportion of crying time (\%) (mean, SD) & $61.19(28.94)$ & $64.21(28.68)$ & $57.86(30.39)$ & $0.628^{\circ}$ \\
\hline Parental VAS score (out of $10 \mathrm{~cm}$ ) (mean, SD) & $3.71(2.56)$ & $3.88(2.40)$ & $3.52(2.85)$ & $0.756^{\circ}$ \\
\hline Did parent think treatment was effective - $\mathrm{n}(\%)$ & $\begin{array}{c}\mathrm{No}=4(19) \\
\text { Yes=11 (52.4) } \\
\text { Uncertain=6 (28.6) }\end{array}$ & $\begin{array}{c}\text { No=3 }(27.3) \\
\text { Yes }=5(45.5) \\
\text { Uncertain=3 (27.3) }\end{array}$ & $\begin{array}{c}\mathrm{No}=1(10) \\
\text { Yes=6 (60) } \\
\text { Uncertain=3 (30) }\end{array}$ & $0.725^{\#}$ \\
\hline Would parent use treatment next time - n (\%) & $\begin{array}{c}\text { No=3 (14.3) } \\
\text { Yes=17 (81.0) } \\
\text { Uncertain=1 (4.8) }\end{array}$ & $\begin{array}{c}\text { No=2 }(18.2) \\
\text { Yes }=9(81.8) \\
\text { Uncertain=0 }(0)\end{array}$ & $\begin{array}{c}\text { No=1 }(10) \\
\text { Yes }=8(80) \\
\text { Uncertain=1 (10) }\end{array}$ & $1.000^{\#}$ \\
\hline Did nurse think child was compliant with treatment - $\mathrm{n}(\%)$ & $\begin{array}{c}\text { No=4 }(19.0) \\
\text { Somewhat }=11(52.4) \\
\text { Fully=6 (28.6) }\end{array}$ & $\begin{array}{c}\text { No=3 }(27.3) \\
\text { Somewhat }=5(45.5) \\
\text { Fully=3 }(27.3)\end{array}$ & $\begin{array}{c}\text { No=1 }(10) \\
\text { Somewhat }=6(60) \\
\text { Fully=3 }(30)\end{array}$ & $0.725^{\#}$ \\
\hline
\end{tabular}

FLACC, Face, Legs, Activity, Cry, Consolability; IQR, interquartile range; SD, standard deviation; VAS, visual analogue scale.*Mann Whitney test; ' ${ }^{\circ}$ Student's t-test; ${ }^{*}$ Fisher's exact test. 


\section{Sample size calculation}

The children in the pilot study cried for a mean of approximately $60 \%$ of the procedure. An absolute reduction of $15 \%$ crying time, down to $45 \%$ of the procedure was considered to be clinically significant. Standard deviation in percentage time crying was $30 \%$, thus a $15 \%$ reduction corresponds to a medium effect size. ${ }^{6}$ Fixing the probability of type I error at $5 \%, 64$ children per study group would provide $80 \%$ power to detect such an effect.

\section{Discussion and Conclusions}

Results confirmed that a full scale RCT is justified, as uncertainty of the effectiveness of sucrose in young children remains. The study interventions were acceptable to children, parents and nurses, the trial was feasible to conduct, recruitment processes, data collection, entry, storage and analysis were all tested and preliminary estimates of treatment effect were gathered and used to perform a sample size estimation. Most children were laid flat during the venipuncture procedure with a median of 3 people holding them. As young children often become fearful and distressed when positioned supine and restrained, upright secure comfortable parental holding during venous access procedures has been promoted, ${ }^{7}$ and will be included as standard of care in the full scale RCT when possible.

Exploring effective pain management for young children undergoing venipuncture is important due to the necessity of the procedure and the high degree of distress demonstrated. This pilot RCT successfully informed the planning of a future full-scale RCT of sucrose for pain management during venipuncture in young children. A full-scale, sufficiently powered RCT will contribute important information to the current body of knowledge concerning pain reduction in young children during painful procedures.

\section{References}

1. Stevens B, Abbott L, Yamada J, et al. Epidemiology and management of painful procedures in hospitalized children across Canada. C Can Med Assoc J 2011;183: E403-10.

2. Kortesluoma RL, Nikkonen M. "I had this horrible pain": the sources and causes of pain experiences in 4- to 11-year-old hospitalized children. J Child Heal Care 2004; 8:210-31.

3. Taddio A, Chambers CT, Halperin SA, et al. Inadequate pain management during routine childhood immunizations: the nerve of it. Clin Ther 2009;31:S152-67.

4. Harrison D, Bueno M, Yamada J, et al. Analgesic effects of sweet tasting solutions in infants: Current State of equipoise. Pediatrics 2010;126:894-902.

5. Harrison D, Stevens B, Yamada J, et al. Sweet tasting solutions for needle-related procedural pain in infants and children aged 1 to 16 years. Cochrane Database Syst Rev 2011;(10):CD008408.

6. Cohen J. Statistical power analysis for the behavioral sciences. $2^{\text {nd }}$ ed. Hillsdale, $\mathrm{NJ}$ : Lawrence Erlbaum Associates; 1988.

7. Sparks LA, Setlik J, Luhman J. Parental holding and positioning to decrease IV distress in young children: a randomized controlled trial. J Pediatr Nurs 2007;22:440-7. 